\title{
A Performance Analysis of the GBN-ARQ Schemes For Satepite Communications
}

M fouad

Abstract

The tandem-type go-back-N (GBN) scheme has been proposed for satellite communications to reduce the long round trip delay using the on-board processing of satellites. This paper presents the tandem-type go-back-N (GBN) scheme, in which the up $\neg$ link and the down link use separate go - back- N ARQ's The performance analysis of the point - to -point tandem (GBN) ARQ and Broadcast tandem type (GBN) ARQ schemes is obtained. The mathematical formulas for the throughput efficiency and the optimal packet 